\title{
EPIDEMIOLOGÍA, ARQUITECTURA PAISAJISTA, «UNA SALUD» E INNOVACIÓN: EXPERIENCIA EN UNA COMUNIDAD AMAZÓNICA
}

\author{
Jorge O. Alarcón ${ }^{1,2, a}$, Jorge A. Alarcón ${ }^{3,4, b}$, Leann Andrews ${ }^{2,3, c}$
}

\begin{abstract}
RESUMEN
Este artículo hace breve referencia a los antecedentes del concepto de «Una Salud» en el contexto mundial y peruano, y la forma como esta perspectiva holística de salud sirvió de fundamento para la creación del Centro de Investigaciones, Tecnológicas, Biomédicas y Medioambientales (CITBM) en la Universidad Nacional Mayor de San Marcos. Seguidamente, describe el proyecto InterACTION Labs, una de las experiencias que usa el enfoque de «Una Salud», liderado por el CITBM, la Universidad de Washington y Traction, esta última una organización no gubernamental enfocada en el diseño e investigación en salud y ecología. InterACTION Labs es un programa de diseño e investigación-acción transdisciplinaria (TDAR, por sus siglas en inglés) que se desarrolla en «Claverito», una comunidad flotante en la ciudad de lquitos en la Amazonía peruana, y que se sustenta en la hipótesis de que el entorno construido es determinante de la salud, tanto del ser humano como de otras especies (aves, reptiles, plantas, etc.). También, discute el concepto de entorno construido en relación a la teoría y práctica de la salud pública, la arquitectura paisajista, la epidemiología y otros campos de la salud y del medio ambiente. Finalmente, reflexiona en los logros y desafíos del proyecto que comenzó en 2015, y describe el plan de trabajo para los próximos años, en los que se propone extender el programa y el uso del enfoque de «Una Salud» para promover la salud humana, ecológica y ambiental en el Perú y otras partes del mundo.
\end{abstract}

Palabras clave: Epidemiología; Iniciativa Una Sola Salud; Ambiente construido; Investigación Participativa Basada en la Comunidad; Investigación Interdisciplinaria (fuente: DeCS BIREME)

\section{EPIDEMIOLOGY, LANDSCAPE ARCHITECTURE, “ONE HEALTH,” AND INNOVATION: EXPERIENCE IN AN AMAZONIAN COMMUNITY}

\begin{abstract}
This article makes brief reference to the background of the "One Health" concept in the global and Peruvian context, and to the way in which this holistic health perspective served as the foundation for the creation of the Center for Technological, Biomedical, and Environmental Research (CITBM) at Universidad Nacional Mayor de San Marcos. Additionally, it describes the InterACTION Labs project, one of the experiences using the "One Health" approach, led by CITBM, the University of Washington, and Traction, an NGO focused on design and research in health and ecology topics. InterACTION Labs is a design and transdisciplinary action-research (TDAR) program developed in "Claverito," a floating community in the city of lquitos in the Peruvian Amazon, based on the hypothesis that the built environment is a health determinant for both humans and other species (birds, reptiles, plants, etc.). It also discusses the concept of 'built environment' as related to the theory and practice of public health, landscape architecture, epidemiology, and other health and environmental fields. Finally, it reflects on the achievements and challenges of the project that began in 2015 , and describes the work plan for the coming years, with a proposal to extend the program and use the "One Health" approach to promote human, ecological, and environmental health in Peru and other parts of the world.
\end{abstract}

Keywords: Epidemiology; One health; Built environment; Community-Based Participatory Research; Interdisciplinary Research (source: MeSH NLM)

\footnotetext{
Instituto de Medicina Tropical de la Universidad Nacional Mayor de San Marcos (UNMSM). Lima, Perú

Centro de Investigaciones Tecnológicas, Biomédicas y Medioambientales (CITBM). Lima, Perú.

Landscape Architecture Department, University of Washington. Seattle, EE.UU.

Traction. Seattle, EE.UU.

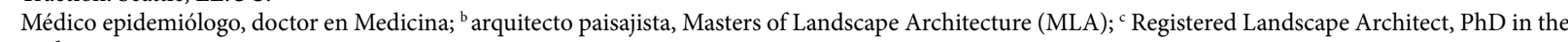
Built Environment

Recibido: 10/12/2018 Aprobado: 12/12/2018 En línea: 21/12/2018
}

Citar como: Alarcón JO, Alarcón JA, Andrews L. Epidemiología, arquitectura paisajista, «Una Salud» e innovación: experiencia en una comunidad amazónica. Rev Peru Med Exp Salud Publica. 2018;35(4):667-74. doi:10.17843/rpmesp.2018.354.4109. 


\section{ENFOQUE HOLÍSTICO DE SALUD E INNOVACIÓN}

En torno a la iniciativa «Una Salud» (One Healh, en inglés) se agrupan varios conceptos cuya discusión se intensificó desde mediados del sigloXIX, momento en el que se expresó con mayor claridad la estrecha relación entre el desarrollo industrial y la salud humana. Notables científicos de aquella época alertaron que era indispensable hacer cambios sociales para combatir las epidemias que afectaban a las poblaciones pauperizadas del campo y de las nuevas urbes surgidas con la creciente migración y sobreexplotación de la fuerza de trabajo de hombres, mujeres y niños. Abundan los libros y artículos que desarrollan este punto de vista, como los artículos de William Farr relacionados al cólera, y que recientemente han vuelto a ser analizados a propósito de las diferencias de opinión con John Snow (1), así como lo que escribió sobre la enfermedad de los mineros (2), los de Rudolf Virchow que, como consecuencia de sus observaciones acerca de la epidemia de tifus en la Alta Silecia concluyó que los cambios climáticos por si solos no explican las epidemias y que para que ocurran es necesario que la gente viva en malas condiciones sociales durante largos períodos de tiempo ${ }^{(3)}$, y los de Godlberger que en su afán por conocer la causa de la pelagra develó el drama social de quienes padecían dicha enfermedad en el sur de los Estados Unidos y que provocó enconadas discusiones acerca de su origen (4).

En Perú, esta discusión se dio con más fuerza entre los años 60 y 70, al mismo tiempo que en América Latina surgía una fuerte corriente que postulaba la relación entre salud y sociedad, y cuestionaba la práctica médica y la formación médica centrada en el hospital, que no presta atención a lo que ocurre con la población en sus propios lugares de trabajo y vivienda. La Organización Panamericana de la Salud acogió muchas de estas ideas y no faltaron expertos que se sumaron a esta corriente, como Juan Cesar García ${ }^{(5)}$. A pesar de estos esfuerzos y del tiempo transcurrido, tanto la práctica de la medicina como la enseñanza han seguido el mismo modelo, centrado en el hospital, más preocupado por la enfermedad que por la salud, por la curación que por la prevención. A los políticos, a su vez, les ha sido más cómodo pensar en la curación que en la prevención, pues pueden ofrecer logros inmediatos sin preocuparse de las consecuencias futuras.

Pero la realidad es tozuda, y nuevos acontecimientos están mostrando a los científicos y políticos la estrecha relación que tiene la salud humana con la de otros seres vivos $y$ con el medio ambiente. La emergencia y reemergencia de las enfermedades infecciosas nos ha enfrentado a nuevos retos, pues su origen no se limita a la presencia de un nuevo germen (que en muchos casos no es nuevo) sino a un conjunto de condiciones que estan interrelacionadas con los cambios climáticos, biológicos y demográficos, y cuyo efecto en la salud, de la que se supone la más exitosa de las especies, ha sido muchas veces catastrófico. Nadie ahora puede ignorar que la vida en el planeta depende del delicado equilibrio entre la salud humana, animal y el medio ambiente ${ }^{(6)}$.

Asistimos, pues, a un proceso en el que la urbanización acelerada, la inequidad social, la degradación del medio ambiente, la extinción de especies, el cambio climático, la exposición a enfermedades y la inseguridad alimentaria y del agua son problemas inextricablemente vinculados. $Y$ es que la vida en el planeta está interrelacionada, de modo que la salud humana, la de otras especies y el ambiente son interdependientes ${ }^{(7)}$. La biodiversidad y las funciones del ecosistema afectan a la salud y bienestar humano a través de los sistemas compartidos de alimentos, agua y suelo, la transmisión de enfermedades, la regulación del clima y las innovaciones farmacéuticas, energéticas y de materiales ${ }^{(8)}$.

Esta situación hace que hoy, a diferencia de los años 60 , nadie discuta la intrincada relación que existe entre el entorno social y ambiental; por el contrario, se han generado movimientos en defensa y cuidado del ambiente, en defensa de la vida animal y en la necesidad de mejorar el entorno en el que viven las poblaciones. Una de estas iniciativas, que cobra cada vez más importancia, es «Una Salud» o «One Health», que en resumen es una perspectiva holística de la salud, que relaciona la salud humana con la salud animal y la salud ambiental ${ }^{(9,10)}$.

Estas ideas acerca de la salud y el medio ambiente fueron el fundamento de la propuesta que la Universidad Nacional Mayor de San Marcos (UNMSM) presentó al concurso que el Consejo Nacional de Ciencia y Tecnología de Perú (CONCYTEC) convocó sobre centros de excelencia el 2014 y que dio lugar a la creación del Centro de Investigaciones Tecnológicas, Biomédicas y Medioambientales (CITBM) con dos líneas de investigación: Biotecnología y salud; y Agua, suelo y sociedad, con la misión de contribuir al bienestar y la salud de las poblaciones y la integridad del medio ambiente a través del desarrollo de tres estrategias: una alianza efectiva y eficiente entre el Estado, la academia y la empresa; un trabajo interdisciplinario y la promoción de una formación integral del científico y de los profesionales. El proceso de desarrollo de esta iniciativa, que lleva casi cuatro años, ha comenzado a proporcionar evidencias acerca de las bondades de este enfoque.

Una de las principales experiencias relacionadas al enfoque «Una Salud», compartida con la Universidad de Washington y diversas instituciones nacionales y extranjeras, es el proyecto InterACTION Labs. Se trata de un programa de investigación-acción transdisciplinario (TDAR, por sus 
siglas en ingles), que se ejecuta con la comunidad flotante de Claverito en la selva amazónica y que incorpora en el proceso de investigación, la participación de la comunidad, el trabajo multidisciplinario, la innovación y la capacitación de investigadores altamente calificados.

\section{EL PROYECTO interACTION Labs, APLICACIÓN DEL ENFOQUE «UNA SALUD» EN UNA COMUNIDAD AMAZÓNICA}

La comunidad de Claverito es un asentamiento humano flotante ubicado a las orillas del centro histórico de la ciudad de Iquitos, Loreto, Perú. Esta comunidad está formada por 52 viviendas flotantes en condiciones precarias, 280 habitantes, 240 animales domésticos y cientos de especies de aves, insectos, reptiles, peces y plantas silvestres. La comunidad no cuenta con energía eléctrica ni saneamiento y tampoco es reconocida en los sistemas de catastro y planeamiento de la municipalidad ni otra entidad del gobierno. La interrelación tan cercana entre la biodiversidad de especies, el ambiente y las condiciones socio económicas extremas de los habitantes provee una oportunidad única para aplicar el concepto de «Una Salud».

En forma resumida, InterACTION Labs primero desarrolló una sólida línea de base de las condiciones ambientales, ecológicas y de salud humana en Claverito, para lo cual impulsó un intenso proceso participativo de la comunidad para definir sus prioridades y los proyectos que desean. Luego, cada año se han implementado los proyectos priorizados, que incluyen: comunidad, hogar y jardines flotantes, escaleras públicas y un centro comunitario. Al mismo tiempo se estudian temas específicos de salud humana y ecológica. Después de la intervención, los investigadores evalúan los cambios en los indicadores de salud, como microbioma humano y ambiental, salud física, oral, mental y social, seguridad alimentaria, diseño, contaminación del agua y de la tierra, riesgo de vectores, biodiversidad, entre otras mediciones. Las Figuras 1 y 2 muestran la situación inicial y los cambios a los dos años del proyecto.

Este programa altamente integrado está entrando en su tercer año y ha involucrado a más de 90 profesionales y estudiantes de 26 campos diferentes, incluyendo salud global, arquitectura paisajista, enfermería, bioingeniería, ciencias ambientales y otros; cinco universidades, incluyendo la Universidad de Washington y la Universidad Nacional Mayor de San Marcos; ocho centros de investigación, incluido el Centro de Investigación en Salud para la Salud $(\mathrm{COHR})$ de la Universidad de Washington y el Centro de Investigación, Tecnológicas, Biomédicas y Medioambientales (CITBM); organizaciones profesionales como la Fundación de Arquitectura de Paisaje (LAF) y la Sociedad Profesional de
Arquitectos en Perú (CAP); e instituciones gubernamentales, incluidos los Institutos Nacionales de Salud de los EE.UU. $(\mathrm{NIH})$, el Instituto de Salud de Perú (INS) y el CONCYTEC.

Esta propuesta de intervención ha tenido la singularidad que surgió desde la perspectiva de un grupo de arquitectos paisajistas acerca de la salud y el ambiente, y que encuentra puntos de contacto con los nuevos enfoques de otras disciplinas, como la epidemiología, la salud pública y las ciencias del medio ambiente. Se sustenta en el concepto de entorno construido (arquitectónico, paisajista, urbanista, etc.), definido como el entorno compartido que ha sido alterado por los seres humanos, y en el que viven, trabajan y se recrean. La Figura 1 muestra la hipótesis explorada en esta investigación, que postula que las mejores condiciones del entorno construido compartido darán como resultado una mejor salud humana y ecológica. Por el contrario, las condiciones ambientales deficientes pueden contribuir a la mala salud humana y ecológica (Figura 3).

\section{EL PAPEL DEL CONCEPTO DE «ENTORNO CONSTRUIDO»EN LA SALUD HUMANA Y ECOLÓGICA}

La idea de que el entorno construido (arquitectónico, paisajista, urbanista, etc.) desempeña un papel importante y multifacético en la salud humana y ecológica, que debe considerarse parte integrante de los sistemas de salud pública, cuenta con el respaldo de un amplio y creciente número de publicaciones científicas. Adicionalmente, estos entornos pueden ser indicadores tanto de la salud humana como ecológica, pues en ellos están impresos los hábitos culturales, las condiciones socioeconómicas, los procesos naturales y el comportamiento de los organismos vivos a lo largo de la historia. Son como cápsulas de tiempo y códigos de barras que expresan la buena o mala salud de sus habitantes ${ }^{(11)}$.

Los entornos construidos se consideran determinantes sociales de la salud que crean circunstancias y oportunidades para obtener resultados saludables, incluso, que podrían ser un componente crítico para mejorar la equidad en la salud ${ }^{(12)}$, así como en la promoción de la salud y la prevención de enfermedades (13). Adicionalmente, investigaciones paralelas, que examinan la salud ecológica en los ambientes construidos, conectan temas ambientales, como la pérdida de biodiversidad y el cambio climático, con los impactos en la salud humana, lo que sugiere que los paisajes ambientales construidos son determinantes de la salud tanto para las especies no humanas como para los humanos ${ }^{(14,15)}$.

Los científicos de la salud pública y del medio ambiente a menudo se refieren a jardines, parques e infraestructura verde dentro de los ambientes construidos urbanos como naturaleza urbana o experiencias cercanas de la naturaleza. Al respecto, hay evidencias científicas que indican vínculos prometedores entre la naturaleza urbana y los impactos 

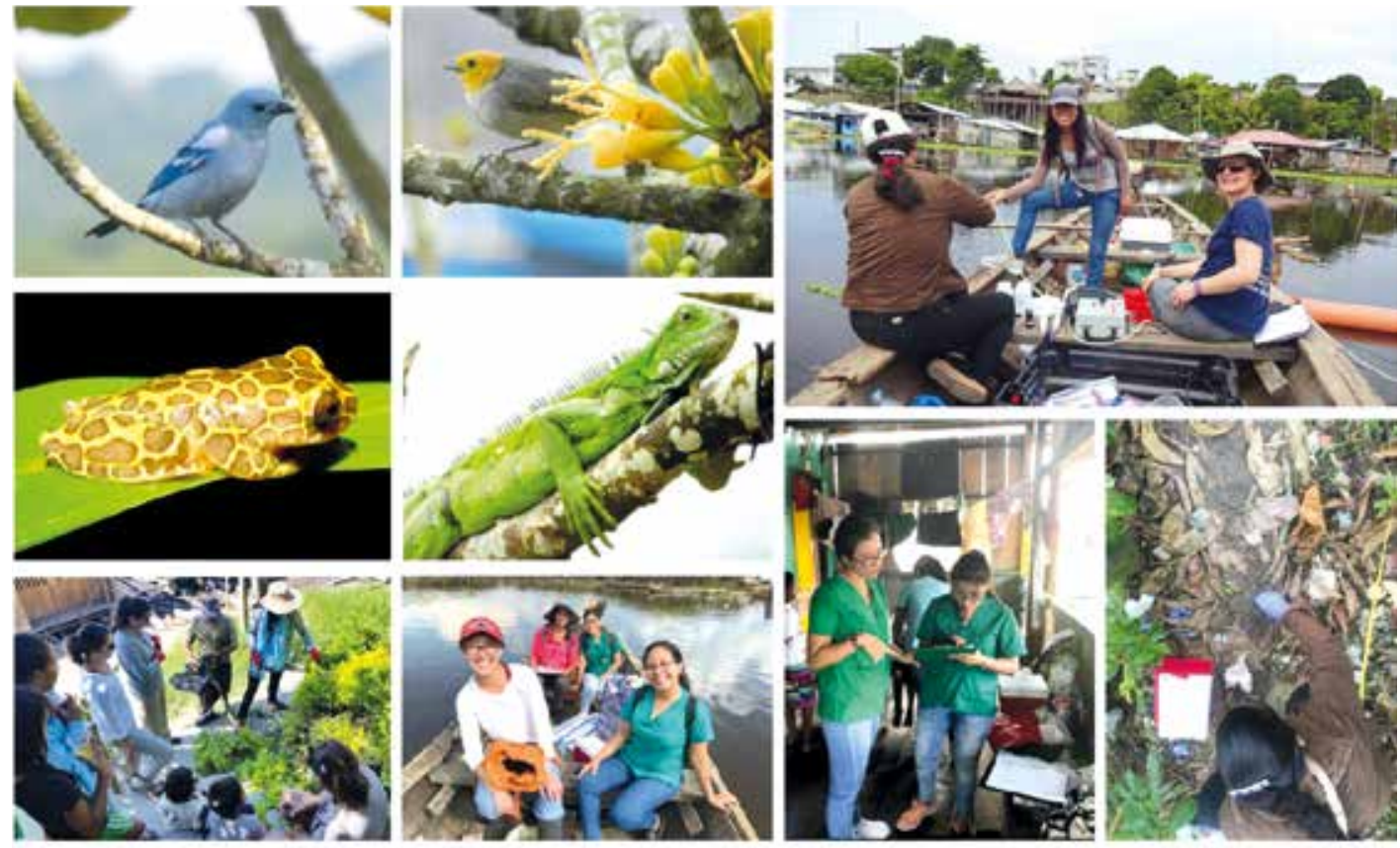

Fuente: Elaboración propia

Figura 1. Evaluaciones para medir condiciones en salud humana, flora, fauna y medio ambiente, y posteriormente medir el impacto de las intervenciones de InterACTION labs con el enfoque transdisciplinario de «Una Salud», realizado por profesionales de 26 distintas disciplinas del «ambiente construido», salud humana y ciencias naturales

positivos y medibles sobre la salud física, mental, social y económica ${ }^{(16-18)}$. La naturaleza urbana puede restaurar funciones del ecosistema; lo cual es importante porque los seres humanos también se benefician de la salud de los ecosistemas y sus habitantes a través de los servicios ecosistémicos que brindan; de otro lado, la pérdida de estos servicios en áreas urbanas densas contribuye a una deficiente salud y bienestar humano ${ }^{(19)}$. También hay una corriente de diseño urbano que busca la promoción de jardines terapéuticos. Estos pueden crear espacios de restauración para poblaciones específicas, desempeñando un papel fundamental en la atención paliativa o la rehabilitación al reducir la gravedad de los síntomas y ayudar a las personas a sobrellevar enfermedades y/o traumas ${ }^{(20)}$. Cuando los jardines se diseñan en respuesta a condiciones ambientales, sociales, políticas, económicas y culturales extremas o difíciles, como ocurre en los barrios marginales, pueden fortalecer otras dimensiones de la salud, como el incremento de la resiliencia y el empoderamiento social de la población ${ }^{(21)}$.

Por su parte, los arquitectos paisajistas, como diseñadores de entornos construidos, han considerado durante mucho tiempo el diseño para la salud humana y ecológica mediante la manipulación del entorno compartido, aunque a menudo no lo manifiestan o reflexionan de manera explícita o crítica. Cuando la teoría miasmática, o la idea de que la «falta de salud» surge del paisaje, fue de conocimiento popular en el siglo XIX, Frederick Law Olmsted, padre de la arquitectura paisajista, diseñó el Central Park en la ciudad de Nueva York con la idea de que también era posible lo contrario: Los entornos construidos podrían ser diseñados para reducir el miasma y, por lo tanto, aumentar la salud ${ }^{(22)}$. La teoría miasmática fue dejada de lado con el descubrimiento de los microorganismos; sin embargo, las especialidades más recientes dentro de la profesión del paisaje, como el diseño de jardines curativos, monumentos y paisajes terapéuticos, aun enfocan el diseño con el objetivo de lograr el bienestar humano, aunque a menudo para propósitos particulares y no colectivos.

En los años 70 y 90, las tendencias en las profesiones de diseño cambiaron hacia «arreglar» los paisajes posindustriales dañados que causaban problemas ambientales y de salud humana, y comenzaron a incorporar datos de salud humana y ecológica al proceso de diseño ${ }^{(23)}$. Más tarde, a mediados de la década del 2000, el diseño paisajista derivó en infraestructura ecológica regenerativa y de multi-rendimiento preventivo, es decir intervenciones ambientales (arquitectónicas, paisajistas, etc.) que tienen como objetivo regenerar y/o proveer uno o varios servicios ecológicos (ciclos energéticos, hábitat, agua, etc.) y otros beneficios para el ser humano y los entornos en los que se construyen. Un ejemplo son los llamados jardines de lluvia diseñados para capturar el agua de lluvia, infiltrar el agua en el subsuelo, limpiar el agua, regenerar el ciclo del agua en las zonas urbanas, proveer hábitat para diversas 

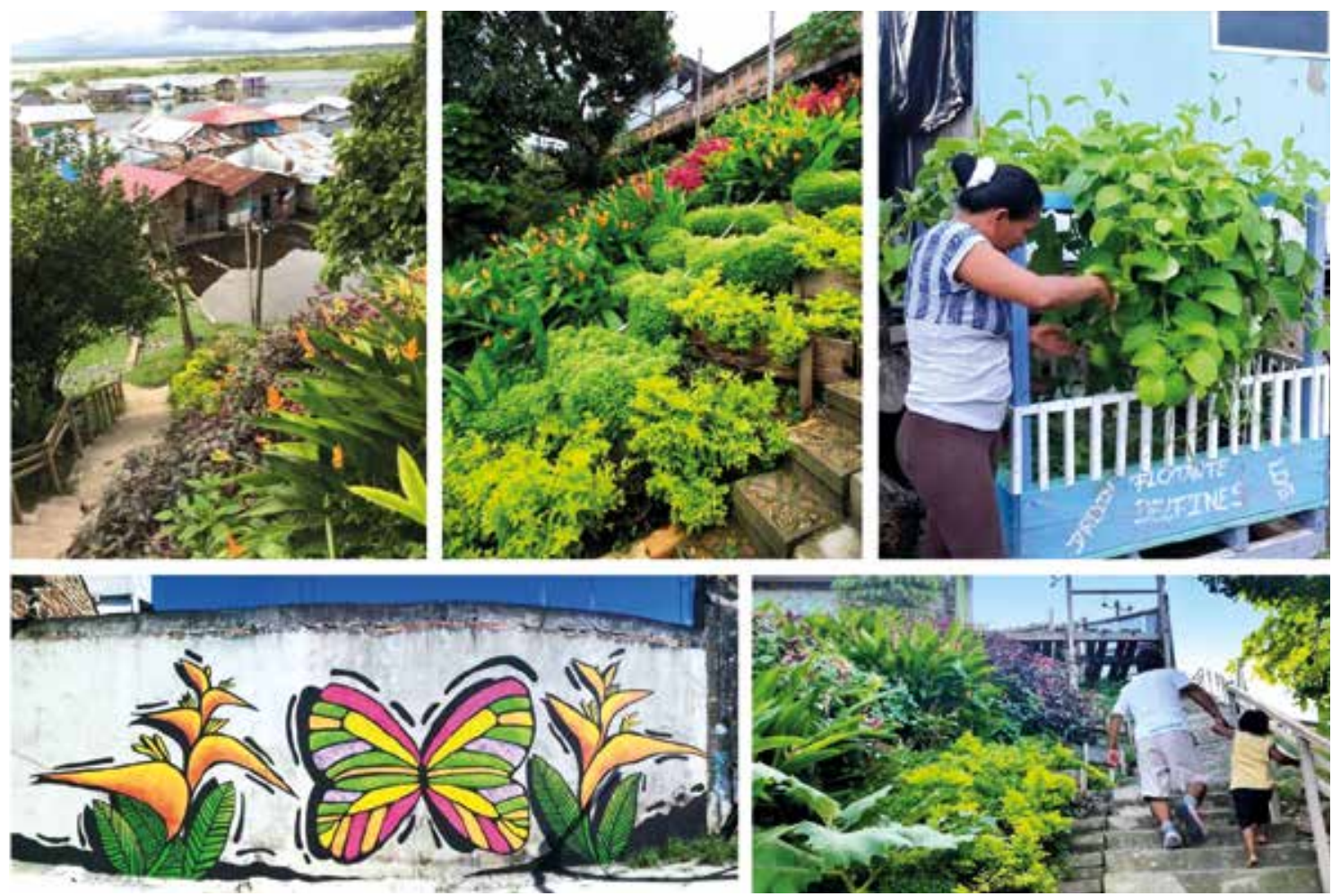

Fuente: Elaboración propia

Figura 2. Ejemplos de intervenciones en el «ambiente construido» del proyecto InterACTION Labs, incluyendo jardines domésticos flotantes, jardines comunitarios, escaleras y arte urbano

especies y ornamentar la ciudad para el beneficio de los usuarios.

Estas nuevas infraestructuras actualmente pueden ser evaluadas con indicadores y certificaciones estandarizadas como LEED (por las siglas en inglés de liderazgo en energía y diseño ambiental) y The Sustainable SITES Initiative (la iniciativa para sitios sostenibles) que cuantifican los logros de los diseños en temas energéticos, ambientales y ecológicos. Recientemente, el término diseño ecológico urbano se usa para referirse a diseños que imitan los sistemas ecológicos para mejorar los ecosistemas y los servicios sociales con la intención de adecuar el entorno construido para cumplir los objetivos de salud humana y ecológica, con lo cual se alinea con la iniciativa de «Una Salud» ${ }^{(23,24) .}$

A pesar del creciente interés en estos nuevos diseños, los arquitectos paisajistas y otros diseñadores del entorno construido no reciben capacitación en su formación académica para entender y abordar los problemas de salud en las comunidades y rara vez trabajan en proyectos que evalúan las condiciones de salud para comprender los impactos de su trabajo y aumentar el conocimiento profesional de su campo ${ }^{(11)}$. De manera similar, los modelos de salud contemporáneos a menudo ignoran las dimensiones ecológicas de la salud humana, y los profesionales de la salud y ecología no reciben capacitación en su formación académica para interactuar con las comunidades y comprender cómo se pueden rediseñar el entorno y/o ambiente físico para maximizar los beneficios para la salud ${ }^{(25)}$.

La urgencia de los problemas de salud ambiental global y sus impactos en la salud deberían inspirar cambios en el enfoque de la investigación y las prácticas de acción comunitaria. Los marcos unidisciplinarios no están equipados para manejar la complejidad de los desafíos de salud humana, ecológica y ambiental que enfrentamos hoy; estos desafíos requieren enfoques integrales para su análisis y el desarrollo de soluciones ${ }^{(26)}$. La necesidad urgente de recopilar datos interdisciplinarios, hacer investigación integrada y desarrollar estrategias de acción colaborativa están llegando a todos los ámbitos del entorno construido, la salud humana y la ecología (27-29). Si bien, el número de publicaciones científicas sobre paisajes de entornos construidos, naturaleza urbana, jardines y salud es abundante y creciente, hay poca investigación exhaustiva que examine sus múltiples impactos aplicados simultáneamente a través de dimensiones humanas, ecológicas y ambientales, debido al predominio de la unidisciplinariedad en la investigación y en el mundo 
a)

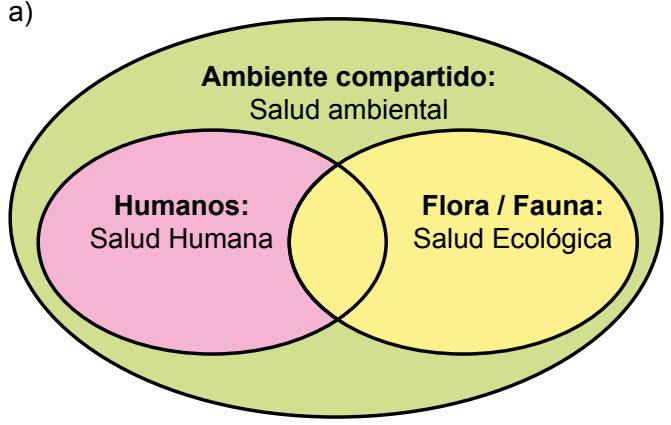

b)

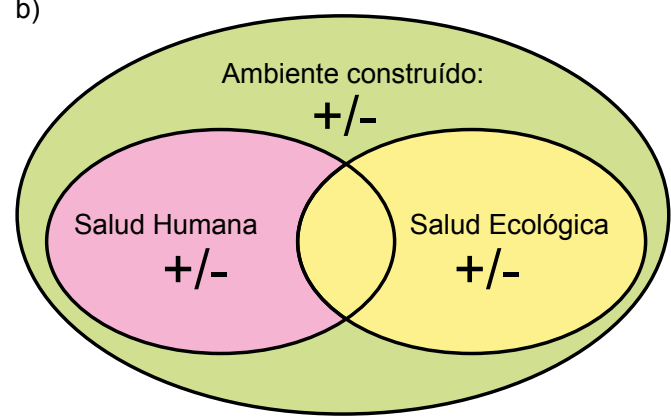

Fuente: Gráfico elaborado sobre la base de la definición de «Una Salud» propuesta por King, et al. (11)

Figura 3. a) La salud humana, la flora, la fauna y el ambiente compartido están relacionados. b) Consecuentemente, mejoras en las condiciones del ambiente construido compartido pueden contribuir a mejorar la salud humana y ecológica; por el contrario, condiciones pobres en el ambiente construido puede contribuir a empeorar la salud humana y ecológica

académico. Por ende, la salud y las preguntas clave siguen sin plantearse ni resolverse ${ }^{(30,31)}$.

La Investigación de Acción Transdisciplinaria (TDAR) es un marco para la investigación de acción colaborativa que cruza diversas disciplinas para abordar problemas sociales y ecológicos complejos de «Una Salud» de manera aplicada ${ }^{(32)}$. TDAR examina la traducción de la investigación y las diversas culturas de conocimiento en estrategias de resolución de problemas de la comunidad mediante la colaboración efectiva con una diversidad de investigadores, miembros de la comunidad, expertos y organizaciones locales ${ }^{(33)}$.

\section{LECCIONES APRENDIDAS Y DESAFÍOS}

Todas estas consideraciones acerca del papel del entorno construido en la salud y el medio ambiente se tomaron en cuenta al diseñar el programa de investigación-acción en la comunidad de Clavarito. Adiferencia de otras intervenciones de salud pública, la propuesta surgió de la reflexión de arquitectos paisajistas identificados con el enfoque TDAR, y comenzó con una propuesta arquitectónica saludable, bajo el supuesto de que ello tendría un impacto en el ecosistema y la salud humana. Luego se fueron incluyendo otros estudios que complementaron el conocimiento del medio ambiente y de la forma de medir el impacto de la intervención. La propuesta dista de ser asistencialista y conformista; es más bien una forma efectiva de fortalecer la autoestima y la resiliencia de estas poblaciones que día a día luchan por generar valor para ellos, sus familias, su comunidad y su ecosistema.

Se trata, pues, de una forma de investigación-acción que no sigue los parámetros de los modelos clásicos de la investigación epidemiológica, y que más bien se acerca a los modelos de investigación de los primeros sanitaristas, como la investigación que condujo la Unidad de Epidemiología Geográfica (Geographic Epidemiology) de la Escuela de Salud Pública de la Universidad Johns Hopkins en el Perú, conjuntamente con investigadores del Instituto de Medicina Tropical de la UNMSM y del INS, entre enero y marzo de 1965 en cuatro poblados representativos de la región amazónica (selva baja), la montaña o selva alta, el altiplano y la costa sur. El equipo de investigación estuvo conformado por científicos de diversas áreas, como médicos epidemiólogos, antropólogos, laboratoristas, entomólogos, ingenieros sanitarios y enfermeras de salud pública ${ }^{(34)}$.

Los autores del estudio le dieron el enfoque denominado epidemiología inclusiva (comprehensive epidemiologic studies), que significa que en un mismo estudio se incluye varias enfermedades y condiciones vistas desde diferentes perspectivas profesionales ${ }^{(35)}$. Este enfoque simplifica la aplicación de los procedimientos de estudio, reduce costos y permite el desarrollo de intervenciones efectivas. Lamentablemente, esta perspectiva se perdió con el tiempo para dar lugar a investigaciones aisladas sin mayor impacto en las poblaciones afectadas.

El programa InterACTION Labs retoma la línea de estos salubristas, en un contexto donde cobran vigencia antiguos conceptos acerca de la salud, que se nutren con nuevas evidencias acerca de la integridad de los problemas de la salud y el medio ambiente, así como la necesidad de trabajar en forma transdisciplinaria. La experiencia en Claverito:

- Muestra que la investigación interdisciplinaria con el enfoque de «Una Salud» es factible en el Perú, que aporta temas de investigación nuevos y relevantes para la comunidad global, y que requiere y genera procesos innovadores en la investigación.

- Ha creado nuevas oportunidades de investigación, en cuanto a temas, financiamiento, capacitación y ejercicio profesional en el Perú y los EE.UU. 
- Ha producido un gran volumen de evidencias científicas para muchas disciplinas simultáneamente y en un plazo relativamente corto.

- Ha creado oportunidades para la interacción de disciplinas que usualmente no están relacionadas. En este proceso se ha logrado un intercambio de métodos de investigación y análisis, así como oportunidades de aprendizaje entre disciplinas.

- Ofrece la oportunidad de documentar hechos relativos a personas, ecología y ambientes de poblaciones marginales que usualmente no se registran.

Sin embargo, la experiencia también ha generado desafíos para los investigadores y centros dedicados a estos estudios, como:

- La necesidad de un léxico que se adapte a las necesidades de cada campo y permita la comunicación interdisciplinaria

- La necesidad de impulsar la innovación en diferentes campos como en los diseños, los métodos y las técnicas.

- Lograr el financiamiento suficiente para todos los componentes de la práctica interdisciplinaria en «Una Salud».

- Idear nuevas estrategias para la diseminación del gran volumen de información que involucra muchas disciplinas y profesiones.

\section{CONCLUSIONES}

InterACTION Labs es una iniciativa que comenzó en 2015 , actualmente se encuentra en su tercer año. En los próximos años, se propone crear y promover programas sostenibles en otras comunidades marginales, sobre la base de fuertes relaciones institucionales, comunitarias y científicas, de modo que sea posible lograr una intervención anual en entornos construidos y medir los cambios en salud humana y ecología a través del tiempo. Asimismo, se propone involucrar nuevos actores, disciplinas e instituciones en la práctica de investigación y capacitación interdisciplinaria, y al mismo tiempo seguir investigando y capacitando en la comprensión y uso de enfoques integrales, como el de «Una Salud», que estudien la relación entre la salud humana, la ecológica y el medio ambiente.

Agradecimientos: Los autores expresan su reconocimiento al Center for One Health Research y a su Director, Peter Rabinowitz; al National Institute of Health (NIH) Fogarty International Center y a Joe Zunt de la Universidad de Washington (UW), que lidera el Northern Pacific Global Health Research Fellows Training Consortium; al Centro de Investigaciones de Recursos Naturales (CIRNA); a la Universidad Nacional de la Amazonia Peruana (UNAP); al Instituto de Medicina Tradicional (IMET); a 100,000 Strong in the Americas; a los departamentos y escuelas de Arquitectura Paisajista, Enfermería, Odontología, Ingeniería Ambiental, y Salud Global de la UW; a la Municipalidad de Maynas, Perú; al Instituto Nacional de Salud, Perú; a la UW Population Health Initiative; al UW Global Innovation Fund, al UW Green Futures Lab, y a la Landscape Architecture Foundation. Sin la colaboración de estas instituciones, sus directivos, profesionales y estudiantes, la experiencia descrita no hubiera sido posible.

Fuentes de financiamiento: Ninguno.

Declaración de conflictos de interés: Los autores no refiere conflictos de interés.

\section{REFERENCIAS BIBLIOGRÁFICAS}

1. Bingham $P$, Verlander $\mathrm{N}$, Cheal $\mathrm{M}$. John Snow, William Farr and the 1849 outbreak of cholera that affected London: a reworking of the data highlights the importance of the water supply. Public health. 2004;118(6):387-94.

2. Buck C, Llopis A, Nájera E, Terris M. El desafío de la epidemiología: problemas y lecturas selecionadas. OPS, editor. Washington: OPS/OMS; 1988:69-73

3. Virchow RLK, Rather LJ. Collected essays on public health and epidemiology: Science History Publications, USA; 1985: 418-446

4. Mooney SJ, Knox J, Morabia A. The Thompson-McFadden Commission and Joseph Goldberger: contrasting 2 historical investigations of pellagra in cotton mill villages in South Carolina. Am J Epidemiol. 2014;180(3):235-44. doi: 10.1093/ aje/kwu134.
5. Garcia JC. Paradigmas para la enseñanza de las ciencias sociales en las escuelas de medicina. Educación Médica y Salud. 1971;5(2):130-48.

6. Miller JM. Implications of the one health paradigm for clinical microbiology. Clin Microbiol Newsl. 2010;32(7):51-6.

7. King LJ, Anderson LR, Blackmore CG, Blackwell MJ, Lautner EA, Marcus LC, et al. Executive Summary of the AVMA One Health Initiative Task Force Report. J Am Vet Med Assoc. 2008;233(2):25961. doi: 10.2460/javma.233.2.259.

8. Morand S, Lajaunie C. Biodiversity and health: Linking life, ecosystems, societies. London: ISTE Press; 2018.

9. Kahn LH. The one-health way. Nature. 2017;453(S47):23-34. doi: $10.1038 / 543547 \mathrm{a}$.

10. One Health [Internet]. World Health Organization; 2017 [citado 4 diciembre
2018]. Disponible en: https://www.who. int/features/qa/one-health/en/

11. Andrews L. Integrating human health, ecology and built environment design: A gardens intervention case study with an informal slum community in the Peruvian Amazon [dissertation]. Seattle (WA): University of Washington; 2018.

12. Williams D, Costa M, Odunlami A, Mohammed S. Moving upstream: how interventions that address the social determinants of health improve health and reduce disparities. J Public Health Manag Pract. 2008;14 Suppl:S8-17. doi: 10.1097/01. PHH.0000338382.36695.42.

13. Dannenberg A, Frumkin H, Jackson R, editors. Making Healthy Places: Designing and Building for Health, Well-Being and Sustainability. Washington DC: Island Press; 2011.

14. Zari M. Ecosystem services analysis in response to biodiversity loss caused by the built environment. Sapiens. 2014;7(1). 
15. Dean J, Van Dooren K, Weinstein P. Does biodiversity improve mental health in urban settings? Med Hypotheses. 2011;76(6):877-80. doi: 10.1016/j. mehy.2011.02.040.

16. Wolf K, Robbins A. Metro nature, environmental health, and economic value. Environ Health Perspect. 2015; 123(5): 390-398. doi: 10.1289/ehp.1408216.

17. Seymour V. The human-nature relationship and its impact on health: A critical review. Front Public Health. 2016;4:260. doi: 10.3389/fpubh.2016.00260.

18. James P, Hart J, Banay R, Laden F. A review of the health benefits of greenness. Curr Epidemiol Rep. 2015;2(2):131-142. doi: 10.1007/s40471-015-0043-7.

19. Millennium Ecosystem Assessment [Internet]. Ecosystems and Human Well-Being: Synthesis. Washington: Island Press; 2005. Disponible en: https://www.millenniumassessment.org/documents/document.356.aspx.pdf

20. Winterbottom D, Wagenfeld A. Therapeutic Gardens: Design for Healing Spaces. Portland: Timber Press; 2015.

21. Helphand KI. Defiant Gardens: Making Gardens in Wartime. San Antonio, TX: Trinity University Press; 2006.

22. Szczygiel B, Hewitt R. Nineteenth Century Medical Landscapes: John H. Rauch, Frederich Law Olmsted, and the Search for Salubrity. Bull Hist Med. 2000;74(4):708-34.

23. Amidon J. Big Nature. In: Tilder L, Blostein B, editors. Design Ecologies: Essays on the Nature of Design. New York: Princeton Architectural; 2010. p. 165-181.

24. Rottle N, Yocom K. Ecological Design. Case Postale, Switzerland: AVA Publishing; 2011.

25. Steingraber, S, Hill, K. Human Health and Design: An Essay in Two Parts. In Hill K, Johnson B, editors. Ecology and Design: Frameworks for Learning. Washington DC: Island Press; 2002. p. 191-214.

26. Wilson EO. Consilience: The Unity of Knowledge. New York: Vintage Books; 1998.

27. Frumkin H. Beyond toxicity: Human health and the natural environment. Am J Prev Med. 2001;20(3):234-40.

28. Johnson BR, Hill K. Introduction: Toward landscape realism. In Johnson BR, Hill K, editors. Ecology and design: Frameworks for learning. Washington DC: Island Press; 2002.

29. Alberti M. Advances in urban ecology: Integrating humans and ecological processes in urban ecosystems. New York: Springer; 2009.

30. Guitart D, Pickering C, Byrne J. Past results and future directions in urban community gardens research. Urban For Urban Green. 2012;11(4):364-73. doi: 10.1016/j.ufug.2012.06.007

31. Frumkin $H$, Bratman G, Breslow $S$, Cochran B, Kahn P, Lawler J, et al. Nature contact and human health: A research agenda. Environ Health Perspect. 2017;125(7):075001. doi: 10.1289/ EHP1663.

32. Stokols D. Transdisciplinary Action Research in Landscape Architecture and Planning. Landscape Jrnl. 2011;30(1):111. doi: $10.3368 /$ lj.30.1.1.

33. Thering S, Chanse V. The scholarship of transdisciplinary action research: Toward a new paradigm for the planning and design professions. Landscape Jrnl. 2011;30(1)6-18. doi: 10.3368/lj.30.1.6.

34. Alarcón JO. Presentación de la Sección Especial de Medicina Tropical. An Fac med. 2012; 73(1):27-8.

35. Buck A, Sasaki T, Anderson R, Hitchcock J, Leigh G. Comprehensive epidemiologic studies of four contrasting Peruvian villages. Geographic Epidemiology Unit. Baltimore, Maryland: The Johns Hopkins University; 1967.

Correspondencia: Jorge O. Alarcon Villafuerte Dirección: Calle José Santos Chocano 199

Bellavista, Callao, Perú

Teléfono: (+511) 996866010

Correo electrónico:jalarconv@unmsm.edu.pe

\title{
Ahora nuestra revista incluye:
}

\author{
Publicación anticipada \\ Compartiendo publicaciones científicas con el ciudadano \\ $\checkmark$ Videos de presentaciones conjuntas del Instituto Nacional de \\ Salud y la Academia Nacional de Medicina \\ Galería fotográfica y videos
}

https://rpmesp.ins.gob.pe 